\title{
Do what is right, not what is easy
}

\author{
Timothy J. Gardner, MD
}

\author{
From the Sydney Kimmel Medical College, Thomas Jefferson University, Philadelphia, Pa; and Christiana Care \\ Health System, Center for Heart \& Vascular Health, Newark, Del. \\ Disclosures: Author has nothing to disclose with regard to commercial support. \\ Received for publication Feb 17, 2017; accepted for publication March 6, 2017; available ahead of print April 8, \\ 2017. \\ Address for reprints: Timothy J. Gardner, MD, Christiana Care Health System, Center for Heart \& Vascular \\ Health, 4755 Ogletown-Stanton Rd, Suite 1003, Newark, DE 19718 (E-mail: tgardner@christianacare.org). \\ J Thorac Cardiovasc Surg 2017;153:1425-6 \\ $0022-5223 / \$ 36.00$ \\ Copyright (C) 2017 by The American Association for Thoracic Surgery \\ http://dx.doi.org/10.1016/j.jtcvs.2017.03.027
}

It is uncertain whether the quotation "Do what is right, not what is easy" can be attributed to a single wise man or has simply become a part of the general discourse. Whatever its origin, this familiar quotation has taken on the gravitas of a golden rule, rather than of ethical principle. To this point, although I found the discussion of professional ethics in the context of the Mycobacterium chimaera heater-cooler device (HCD) contamination interesting, the more important takeaway from this essay by $\mathrm{Dr}$ Nguyen and colleagues ${ }^{1}$ is how the Mayo Clinic chose to deal with their patients who were exposed to the $M$ chimaera infection risk. A brief review is presented on what we have learned from this occurrence of nontuberculous mycobacterial (NTM) infections in patients undergoing cardiac surgery.

1. In most of these reported infections in the United States and Europe, the NTM infection has been $M$ chimaera, a slow-growing and virulent organism.

2. M chimaera, like many other mycobacteria, is ubiquitous in the environment and found in public water sources. Although NTM exposure usually is harmless, serious infections do occur, albeit it rarely, as has become evident in patients undergoing cardiac surgery.

3. The HCD, in particular the commonly used Sorin, now LivaNova PLC (London, UK), Stockert 3T HeaterCooler System, has been identified by the Food and Drug Administration (FDA) and Centers for Disease Control and Prevention (CDC) as the likely source of $M$ chimaera infections in the United States.

4. The Sorin HCDs, used for many years in $60 \%$ or more of US cardiac surgery operating rooms, are believed to allow for aerosolized contamination of the operating room environment with $M$ chimaera despite appropriate device cleaning and use.

5. Although the full scope of these $M$ chimaera infections remains unclear, it appears that few patients become infected. It also is unclear whether these infections are clustered in only a few surgical centers or may be discovered more widely in any surgical program using the Stockert 3T HCD. gical procedure.

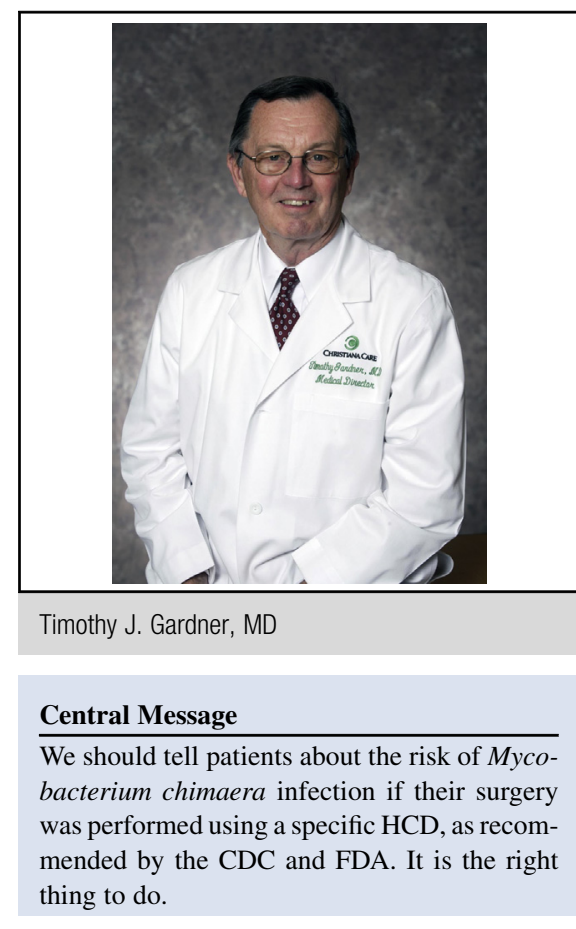

See Article page 1422 .

Because of these events, many cardiac surgery programs have replaced the Stockert 3T HCD with other devices. More thorough cleaning and disinfection of HCDs, use of sterile water instead of public water, controlled venting, and other techniques to mitigate the infection risk with use of HCDs have been widely adopted. This article by Nguyen and associates ${ }^{1}$ at the Mayo Clinic addresses the patient notification challenge that cardiac surgeons and our programs have had to address. Both the FDA and the CDC recommended in 2016 that hospitals notify patients about this infection risk and "establish patient surveillance in cases of potential exposure." ${ }^{2,3} M$ chimaera is a slowgrowing infection that may become apparent only months or years after the cardiac surgery exposure. Patient notification and follow-up "surveillance" pose immense logistic challenges. The important first step in response to this newly recognized infection risk for patients undergoing cardiac surgery is as broad notification as possible of patients who were exposed to the Stockert 3T HCD during their sur-

Whether there is an "ethical duty" to follow the FDA and CDC recommendations to disclose this information to patients was Nguyen and colleagues' ${ }^{1}$ rationale for this article. I believe that it would be unwise for cardiac surgery 
programs that used the Stockert 3T HCD to omit patient notification about this previously unknown infection risk. Both the at-risk patients and the medical community at large should be made aware of the $M$ chimaera risk. This is especially important in this situation because the associated illness may occur so late after the incident surgery. The notification letters sent by the Mayo Clinic to former patients from a 4-year potential exposure range are excellent. The texts of these letters appear in the article's "Appendix." ${ }^{1}$ In addition to general patient notification that is recommended by the FDA and CDC, information about this newly identified infection should be shared widely by health systems with referring physicians, primary care providers, and infectious disease specialists. It should be noted that patients with valve prostheses, grafts, and other devices may be particularly susceptible to late $M$ chimaera infections.

Kudos to the Mayo Clinic for its effective response to the $M$ chimaera infection risk in response to the public alerts from the FDA and CDC. Although the Mayo team reflected on the ethical considerations for this information, it does not require an ethicist to determine that disclosure is the right thing to do in this situation. It is possible, perhaps even likely, that additional cases of late $M$ chimaera infection will be diagnosed. In the meantime, we and our physician colleagues should be alert to the occurrence of this late-developing illness in any patients who underwent cardiac surgery and were exposed to the Stockert 3T HCD.

\section{References}

1. Nguyen A, Christopher Hook C, Dearani JA, Schaff HV. Mycobacterium chimaera: The ethical duty to disclose the minimal risk of infection to exposed patients. J Thorac Cardiovasac Surg. 2017;153:1422-4.

2. US Food \& Drug Administration. UPDATE: Mycobacterium chimaera infections associated with LivaNova PLC (formerly Sorin Group Deutschland $\mathrm{GmbH}$ ) Stöckert 3T Heater-Cooler System: FDA safety communication. Available at: http://www.fda.gov/MedicalDevices/Safety/AlertsandNotices/ucm520191.htm. Accessed October 13, 2016.

3. Centers for Disease Control and Prevention. Contaminated devices putting openheart surgery patients at risk. CDC encourages hospitals to take action, advises patients to seek care if ill. Available at: https://www.cdc.gov/media/releases/2016/ p1013-contaminated-devices-.html. Accessed October 13, 2016. 